\title{
EINE FORM DES 'UNEIGENTLICHEN' SPRECHENS: DIE IRONIE
}

\author{
EKKEHARD EGGS
}

\section{PROBLEMSTELLUNG/FORSCHUNGSLAGE}

In der neueren linguistischen Literatur wird Ironie oft im Zusammenhang mit Witz, Humor, Spott, dem Komischen usw. behandelt. So kommt STEMPEL in seiner Untersuchung "Ironie alsSprechhandlung" zu folgendem Ergebnis: Ziel der ironischen Handlung sei die "(lächerlich machende) Bloßstellung " einer bestimmten Person oder Institution, wobei deren Lächerlichkeit dem Zuhörer mit "komischer Wirkung vermittelt wird «. ${ }^{1}$ Um das an einem Beispiel aus Frevds "Der Witz und seine Beziehung zum Unbewußten" zu verdeutlichen, auf das sich auch STEMres in seiner Untersuchung bezieht:2

(1) "Der König besuchte in seiner Herablassung die chirurgische Klinik und trifft den Professor bei der Vornahme der Amputation eines Beines, deren einzelne Stadien er mit lauten Äußerungen seines königlichen Wohlgefallens begleitet. "Bravo, bravo, mein lieber Geheimrat». Nach vollendeter Operation tritt der Professor an ihn heran und fragt, sich tief verneigend: "Befehlen Majestät auch das andere Bein?"

Opfer - so die Interpretation STEMPELs - Opfer der Ironie ist hier der König, Zuhörer entweder jemand, der diese Szene unmittelbar erlebt oder auch sie alle, denen ich eben diese Geschichte erzählt habe. Thnen habe ich - so die Auffassung Stemrets - die Lächerlichkeit des Königs mit komischer Wirkung vermittelt. Diese Konzeption der Ironie entspricht der in den meisten literaturwissenschaftlichen Abhandlungen ${ }^{3}$ zur Ironie vertretenen Meinung, die besagt, daß ein Protagonist in der Erzählung oder im Drama in irgendeiner Weise lächerlich gemacht und bloßgestellt wird - der Leser oder Zuschauer folgt dieser humorvollen Darstellung mit einem gewissen wissenden Schmunzeln.

Freilich: diese Konzeption kann nicht alle Erscheinungsformen der Ironie bestimmen, weil sie nicht zwischen erzählter Situation und Erzählsituation unterscheidet. Es ist nämlich etwas anderes, 
ob ich meinem Zuhörer gegenüber eine ironische Handlung vollziehe, oder ob ich eine Geschichte erzähle, in der ein Protagonist gegenüber einem anderen Protagonisten sich ironisch verhält. Kurz: zwischen 'ironischer Handlung' und 'Witz oder Geschichte, die eine ironische Handlung zum Gegenstand haben' muß streng unterschieden werden. Genau diese Differenz wird von STEMPEL nicht gesehen. Das hat weitreichende Konsequenzen:

Ein Witz ist zwar dann gut und erfolgreich erzählt, wenn er beim Zuhörer Lachen erzeugt, aber: dieser Lacheffekt ist kein notwendiges Moment der ironischen Handlung. Dies sei noch am IronieModell, das WeINRICH in seiner "Linguistik der Lüge" entwickelt hat, verdeutlicht:

WeINRICH exemplifiziert sein 'elementares Ironiemodell' an der Sokratischen Ironie, genauer: an Platons Dialog: "Euthyphron". Euthyphron hält sich für den besten Kenner in religiösen Fragen. Sokrates' Strategie in diesem Dialog - und das gilt auch für den größten Teil der sokratischen Dialoge - besteht nun darin, bei der Behandlung der Frage, was denn das Fromme sei, den Euthyphron in Widersprüche zu verwickeln und zwar derart, daß Euthyphron am Ende das Gegenteil dessen zugestehen muß, was er ursprünglich behauptet hat. ${ }^{4}$

WEINRICH interpretiert nun die Tatsache, daß Sokrates die Schülerrolle einnimmt und sich von Euthyphron beraten lassen will, ${ }^{5}$ im Laufe des Dialogs aber zeigt, daß er 'mehr' als Euthyphron weiß, dahingehend, daß sich Sokrates unwissend stellt und nur zum Scheine so tut, als ob er nichts wisse. WEINRICH betont dann:

"So läßt sich Sokrates zum Schein belehren, damit der Lehrende an den bohrenden Fragen des Belehrten selber merkt, wie schlecht es um seine Lehre steht und wie er selber der Lehre bedarf (. . .). Es erweist sich, daß das Nichtwissen des Philosophen tatsächlich eine Verstellung war, ein Kleintun. $\|^{6}$

WEINRICH nimmt nun weiter an, daß Sokrates sein ironisches Sprechen durch ein Ironiesignal zum Ausdruck bringt - und zwar durch eine besondere Intonation, nämlich durch eine "zum Salbungsvoll-Bedeutsamen hin verstellten Stimme».7 Dieses Ironiesignal - so die weitere Interpretation WEINRICHs - kommt bei Euthyphron nicht an. Mithilfe dieser Annahmen kann dann WeINRICH sein 'elementares' Ironiemodell wie folgt bestimmen:

"Die sprachlichen Mitteilungen, die von Sokrates ausgehen, gehen demnach in zwei verschiedene Richtungen. Sie spalten sich gleichsam; eine Informationskette geht zum angesprochenen Hörer und sagt Ja, wäh- 
rend eine zweite, begleitende Informationskette zu einem mitangesprochenen Dritten geht und Nein sagt. Diese Informationskette setzt sich aus den Ironiesignalen zusammen.. ${ }^{8}$

Die erste Person ist nach WenNRICH Sokrates, die zweite Euthyphron und die dritte entweder der Zuhörer Platon oder wir als Leser dieses Dialogs.

WeINRICH setzt somit in seinem Ironiemodell drei Personen an, wobei das Opfer der Ironie (hier: Euthyphron), uns als Lesern gegenüber mit 'humorvollem Effekt' lächerlich gemacht wird, ohne daß Euthyphron dies bemerkt.

Freilich: dieses 'elementare' Ironiemodell stellt mehrere Fragen. Zunächst: WeINRICH bestimmt die ironische Handlung nicht als eine spezifische Beziehung der unmittelbar am Gespräch Beteiligten, sondern als eine Beziehung zwischen den Gesprächsteilnehmern und einem Zuhörer oder Leser, der an diesem Gespräch nicht unmittelbar beteiligt und engagiert ist, kurz: WEINRICH bestimmt die ironische Handlung nicht intra-, sondern extrakommunikativ. Deshalb vermischt auch dieses Modell 'erzählte Wirklichkeit' und 'Erzählsituation'. Aus dieser Vermischung folgt zweitens die WeINRICHsche Unterstellung, daß Sokrates die Intention hatte, den Euthyphron bloßzustellen und lächerlich zu machen.

Nun ist die Frage, ob Sokrates 'wirklich' die Absicht hatte, seine Gesprächsteilnehmer ironisch bloßzustellen, unausgemacht. So stellt etwa der späte HEGEL fest:?

"Sonach muß uns jene als unwahr angesehene Einleitung des Sokrates, daß er versicherte, nichts zu wissen, keine Wissenschaft zu besitzen, vielmehr als ganz ernst von ihm gesagt, für ganz richtig und keineswegs für ironisch gelten; wir finden sie durch sein wirkliches Leben und Treiben nicht widerlegt.»

Diese beiden konträren Ansichten über die Frage, ob Sokrates selbst ironisch sein und handeln wollte oder nicht, können ein"weiteres Problem verdeutlichen, nämlich: daß wir oft jemandem eine ironische Absicht zuschreiben, ohne daß dieser ironisch sein oder handeln wollte. Dies ist natürlich nicht nur ein Problem der linguistischen Analyse von ironischen Handlungen, sondern auch ein zentrales Problem der Interpretation von literarischen Werken. So kann man etwa der Ansicht sein, daß Ulrich in Musins Roman Der Mann ohne Eigenschaften ein durch und durch ironischer Mensch ist. Diese Ansicht ließe sich durch viele Stellen wie etwa die folgende abstützen: 
"So war es einmal bei einer Ausfahrt über Land vorgekommen, daß der Wagen an entzückenden Tälern vorbeirollte, zwischen denen von dunklen Fichtenwäldern bedeckte Berghänge nahe an die Straße herantraten, und Diotima mit den Versen "Wer hat dich, du schöner Wald, aufgebaut so hoch da droben . . . ? « darauf hindeutete; sie zitierte diese Verse selbstverständlich als Gedicht, ohne den dazugehörigen Gesang auch nur anzudeuten, denn das wäre ihr verbraucht und nichtssagend erschienen. Aber Ulrich erwiderte: "Die Niederösterreichische Bodenbank. Das wissen Sie nicht, Kusine, daß alle Wälder hier der Bodenbank gehören ? Und den Meister, den Sie loben wollen, ist ein bei ihr angestellter Forstmeister. Die Natur ist hier ein planmäßiges Produkt der Forstindustrie; ein reihenweise gesetzter Speicher der Zellulosefabrikation, was man ihr auch ohne weiteres ansehen kann."10

Wahrscheinlich sind viele Leser dieses Romans geneigt, jemandem, der auf diesen sublim-elegischen Ausruf: "Wer hat dich, $d u$ schöner Wald, aufgebaut so hoch da droben ...?" trocken antwortet: "Die Niederösterreichische Bodenbank...", eine ironische Absicht zuzuschreiben. Freilich wird man bei einer 'genauen' und 'behutsamen' Lektüre des ganzen Romans (wie auch der Äußerungen Musils über seinen Roman $)^{11}$ eher feststellen müssen, daß dieser Ulrich kein Ironiker ist, sondern vielmehr jemand, der ernsthaft nach einer anderen Wahrheit als der herrschenden sucht.

Der bisherige Problemaufriß sollte drei Punkte verdeutlichen:

(i) Der Lacheffekt ist kein notwendiges Moment der ironischen Handlung.

(ii) Die ironische Handlung muß intrakommunikativ bestimmt werden.

(iii) Diese Bestimmung muß die Frage klären, wann und unter welchen Voraussetzungen wir zurecht jemandem eine ironische Absicht zuschreiben können.

\section{DIE IRONISCHE SPRECHHANDLUNG}

Die genannten Punkte seien im folgenden noch weiter begründet. Meine Vorgehensweise möchte ich anhand der Definition (2) kurz verdeutlichen:

(2) "Eh bien, c'est réussi!", dit-il avec ironie devant son travail gâché = raillerie qui consiste à exprimer, sur un ton particulier, le contraire de ce que l'on veut dire.12

Im folgenden werde ich weiter begründen, daß die in dieser Definition genannte 'raillerie' oder 'lächerlich machende Bloßstellung' kein notwendiges Bestimmungsstück der Ironie ist; ich werde zweitens versuchen nachzuweisen, daß zur Realisierung der Ironie 
keine besonderen Ironiesignale notwendig sind (wie dies in der Definition (2) durch die Parenthese: "sur un ton particulier " behauptet wird); und ich werde drittens versuchen, die Bedeutung des Teilsatzes: "Das Gegenteil dessen ausdrücken, was man sagen will" zu präzisieren.

Das Gegenteil dessen ausdrücken, was man sagen will ist offensichtlich nicht gleichzusetzen mit: Das Gegenteil dessen ausdrücken, was man denkt, denn dies würde ja auch für die Lüge gelten. Was ist also die spezifische Differenz zwischen Lüge und Ironie? Eine Antwort auf diese Frage gibt die auf QUINTIIIAN zurückgehende Definition der Encyclopédie; danach ist die Ironie:

(3) Une figure par laquelle on veut faire entendre le contraire de ce qu'on dit. ${ }^{13}$

Also: die Ironie ist eine Redefigur oder Redeweise, bei der man das Gegenteil dessen, was man sagt, zu verstehen geben will. Das ist auch das unterscheidende Merkmal von Ironie und Lüge: der Lügner darf nicht zu erkennen geben, daß er selbst seine Äußerung für falsch hält, der Ironiker hingegen muß dem andern 'irgendwie' mitteilen, daß er das Gegenteil dessen, was er sagt, meint. KrERKEGAARD hat das prägnant formuliert: von einer ironischen Rede

isagt man wohl: 'der Ernst ist nicht ernst gemeint'. Die Äußerung klingt ganz schauderhaft ernst, aber der kundige Hörer ist in das Geheimnis eingeweiht, das dahintersteckt .14 $^{14}$

Damit stellen sich zwei Fragen:

(i) Wie weiht der Ironiker seinen Zuhörer in sein Geheimnis ein? Anders gesagt: mit welchen 'Mitteln' teilt ihm der Ironiker mit, daß er das Gegenteil des Gesagten meint? Und:

(ii) Was heißt das genau: das Gegenteil des Gesagten meinen?

Um mit der zweiten Frage zu beginnen. Sie wurde in klassischen, aber auch neueren rhetorischen Abhandlungen wie folgt beantwortet: ${ }^{15}$ Die Ironie wurde in diesen Abhandlungen immer als tropische Figur im Zusammenhang mit der Metapher behandelt. 'Trope' oder 'tropische Figur' meint, daß in einem bestimmten Kontext ein Term oder eine Phrase verwendet werden, die gewöhnlich in diesem Kontext nicht verwendet werden. Also etwa die Metapher: "Asche stand ihm im Gesicht» statt: "Trauer stand ihm im Gesicht». Legitimiert ist diese Ersetzung durch eine gewisse Bedeutungsähnlichkeit der beiden Terme. Bei der Ironie hingegen - so die rhetorische Theorie - wird nun nicht ein Term durch einen anderen Term 
mit ähnlicher Bedeutung ersetzt, sondern durch einen Term mit antonymer oder konträrer Bedeutung. Also etwa im Beispiel (2): gâché wurde in "Eh bien, c'est réussi !" durch sein Antonym réussi ersetzt. Ebenso ist im bekannten Beispiel der Rede des ANTonius der Satz: "Und Brutus ist ein ehrenwerter Mann" im Sinne von: "Und Brutus ist ein verachtenswerter Mann" zu verstehen.

Gemeinsam ist somit der Metapher und der Ironie, daß etwas durch etwas anderes ersetzt wird; sie unterscheiden sich dadurch, daß im Falle der Metapher diese Ersetzung durch Ähnlichkeit, im Falle der Ironie aber durch Gegensätzlichkeit gesteuert wird.

Nun kann man leicht nachweisen, daß Ironie nicht auf einfache Gegensätzlichkeit reduzierbar ist (und $d . h$. auch, daß die Ironie fundamental von der Metapher verschieden ist). Um das am folgenden Beispiel zu verdeutlichen:

(4) Als die Bewohner von Tarragona Augustus berichteten, daß eine Palme auf seinem Altar gewachsen sei, antwortete dieser: "Ich sehe wohl, daß ihr oft Feuer auf meinem Altar anzündet«.16

(Um dieses Beispiel zu verstehen, muß man wissen, daß die Römer ihre Götter - und dazu zählten auch die Kaiser - ehrten, indem sie auf deren Altären Feuer anzündeten).

Offensichtlich wurde in der Antwort des Augustus kein Term durch sein Antonym ersetzt, sondern: Augustus behauptet etwas als wahr, von dem er selbst weiß, daß es falsch ist, und von dem er weiß, daß die Einwohner von Tarragona wissen, daß es von ihm nicht wahr gemeint sein kann. Anders gesagt: bei einer ironischen Handlung geht es nicht um die Ersetzung von Termen oder Phrasen, sondern um die Stellung des Sprechers zu seiner Äußerung.

Das muß noch weiter präzisiert werden. Die Richtung wird durch die KIERKEGaARDsche Formel: "Der Ernst ist nicht ernst gemeint" angezeigt. Sprachliches Kommunizieren heißt ja nicht nur, daß man sich an sprachliche Konventionen hält (also etwa, daß man sich an Aussprache-, Syntax- und Bedeutungsregeln einer Sprache hält); sprachliches Kommunizieren heißt auch, daß man sich an die Metakonvention der Aufrichtigkeit hält, d.h. daß man die Äußerungen, die man vollzieht, auch aufrichtig vollzieht. ${ }^{17}$ Also etwa: eine Behauptung aufrichtig vollziehen heißt: das Behauptete für wahr halten und geeignete Beweise für die Wahrheit seiner Behauptung haben; oder: ein Versprechen aufrichtig geben heißt: die Absicht haben, die versprochene Handlung auch auszuführen; oder schließlich: eine Frage aufrichtig stellen heißt: seine Intention zum Ausdruck bringen, vom Hörer die erfragte Information zu bekommen. 
Ich bezeichne nun Sprechakte, in denen sich der Sprecher an die sprachlichen Konventionen hält, als DIREKTE SPRECHAKTE. 'Sprachliche Konventionen' meint hier doppeltes: (i) daß der Sprecher sich sprachlich 'so wie gewöhnlich' ausdrückt, d.h. daß er die Wörter in ihrer normalen und gewöhnlichen Bedeutung verwendet; wie auch, daß er normale und gewöhnliche Sätze bildet; 'Sprachliche Konventionen' meint aber auch (ii), daß der Sprecher seine Äußerungen normal situiert, d.h. daß er seine Äußerungen situationsadäquat vollzieht. NICHT-DIREKTE Sprechakte, die gegen die erste Klasse von Konventionen verstoßen, bezeichne ich als RHETORISCHE Sprechakte; Sprechakte, die die zweite Klasse von Konventionen verletzen, bezeichne ich als INDIREKTE Sprechakte. ${ }^{18} \mathrm{Um}$ dafür einige Beispiele zu geben: wenn ich sage: "Das glaub' ich dir nicht», so vollziehe ich einen direkten Sprechakt; drücke ich den gleichen Sachverhalt aber aus durch: "Les jaunes flèches de tes regrets ne me touchent point", so vollziehe ich einen nicht-direkten, genauer: einen rhetorischen Sprechakt.

Ebenso ist etwa die Äußerung: "Es ist schon Mitternacht» situativ normal verwendet, wenn die Satzbedeutung mit der vom Sprecher in der Äußerungssituation gemeinten Bedeutung zusammenfällt d. h., wenn es sich um die Feststellung der Uhrzeit handelt - und um nichts anderes. Wenn ich aber: "Es ist schon Mitternacht" äußere, um meinen Gästen 'durch die Blume' indirekt zu sagen: "Sie sollten jetzt endlich nach Hause gehen - es ist schon Mitternacht", so vollziehe ich einen indirekten Sprechakt; und dieser Sprechakt ist genau dann erfolgreich, wenn meine Gäste mich verstehen und zügig nach Hause gehen.

Nun können offensichtlich alle drei Klassen von Sprechakten aufrichtig und unaufrichtig vollzogen werden. Anders gesagt: Sprecher können sich an die Metakonvention der Aufrichtigkeit halten oder auch nicht. So kann der direkte Sprechakt: "Ich war gestern krank" aufrichtig und unaufrichtig vollzogen sein; er ist aufrichtig vollzogen, wenn es wahr ist, daß ich gestern wirklich krank gewesen bin, sonst ist er unaufrichtig vollzogen oder: eine Lüge. Genauso gelogen hat ein Sprecher, der "Les jaunes flèches de tes regrets ne me touchent point« äußert, wenn - wie man so sagt - er in seinem tiefsten Innern durch die Klagen des andern aufgewühlt ist. Um noch ein Beispiel für einen indirekten Sprechakt zu geben: wenn ich sage: "Der Film X ist ganz toll", um meinen Gesprächspartner indirekt aufzufordern, sich diesen Film anzuschauen, so habe ich mit 
einer Feststellung konversationell eine Aufforderung impliziert. Und wenn ich - ohne Hintergedanken - will, daß der andere sich diesen Film ansieht, dann habe ich einen aufrichtigen indirekten Sprechakt vollzogen. Wenn ich aber diesen Sprechakt vollziehe, um meinen Gesprächspartner dazu zu bringen, ins Kino zu gehen, um ihn während seines Aufenthaltes im Kino zu bestehlen, so habe ich unaufrichtig gehandelt, ja man kann sogar sagen, daß ich ihn damit geschickt betrogen und belogen habe.19

Die gegebene Klassifikation von Sprechakten erlaubt uns nun, das wesentliche Bestimmungsstück derironischen Handlung kurz zu benennen: die ironische Handlung ist unaufrichtig und zugleich aufrichtig vollzogen; der Ironiker tut so, als ob er sich an die Metakonvention der Aufrichtigkeit hält und zeigt dem Zuhörer doch zugleich, daß er diese Metakonvention durchbricht. Dieses 'ernste Spiel' mit der Metakonvention der Aufrichtigkeit sei durch (5) illustriert:

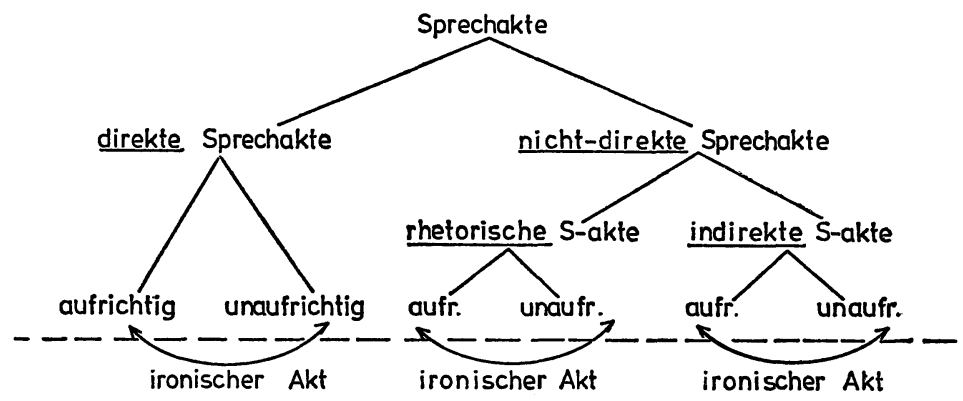

Da der Ironiker seinem Zuhörer zu verstehen gibt, daß er 'unaufrichtig' spricht, ist er - auf einer höheren Stufe - wiederum 'aufrichtig'. Deshalb konnte JANKÉLÉvITCH in seiner Untersuchung "L'ironie ou la bonne conscience" auch schreiben: "L'ironie est encore plus sérieuse que le sérieux $\|^{20}$ Und weil der Ironiker uns sagt: "Der Ernst ist nicht ernst gemeint", bewundern wir ihn auch in bestimmten Hinsichten, vor allem aber dann, wenn er bestimmte Leute in ihrer 'bonne conscience', d. h. in ihrer behäbig-beharrlichen Selbstzufriedenheit entlarvt. Auf die Tatsache, daß wir den Ironiker in anderen Hinsichten ablehnen können, gehe ich weiter unten noch ein. Hier geht es mir nur darum, hervorzuheben, daß Ironie sprachliches Handeln in seiner ganzen Breite betrifft; m.a.W.: ironische Handlungen können mithilfe aller Sprechakttypen vollzogen werden. 
Um das noch an einigen Beispielen zu verdeutlichen: ein Beispiel für eine mithilfe eines direkten Sprechaktes vollzogene ironische Handlung ist ANTONIUS': "Und Brutus ist ein ehrenwerten Mann". Aufgrund des Kontextes wissen oder erfahren die unmittelbaren Zuhörer oder auch wir als Zuschauer, daß ANTonIUs nicht das, was er sagt, zu verstehen geben will. Entsprechend ist im oben gegebenen Quintilianbeispiel (4) die wirkliche und aus dem Kontext erschließbare Meinung des Augustus nicht: "Ich sehe wohl, daß ihr oft Feuer auf meinem Altar anzündet», sondern (sinngemäß umschrieben): "Ich sehe, daß ihr nie/selten Feuer auf meinem Altar anzündet«. Nun kann man nicht nur behaupten, daß ein bestimmter Sachverhalt faktisch besteht, sondern auch den Grund für das Bestehen eines Sachverhaltes behaupten. Auch hier ist Ironie möglich. Ein Beispiel dafür ist etwa:

(6) Ein Gaskogner erwidert auf eine ihn unglaublich dünkende Erzählung nur: "Aber ich werde ihre Geschichte nicht weitergeben, wegen meines Dialekts».21

Um diesen ironischen Witz, genauer: um die ironische Handlung des Gaskogners zu verstehen, muß man wissen, daß der Gaskogner in der französischen Literatur seit dem 16. Jahrhundert als 'Prahlhans' und 'Lügner', den man zudem leicht an seinem Akzent erkennen kann, abqualifiziert wurde (was wiederum die herrschende Meinung über den Gaskogner beeinflußt hat - das hat sich auch im Wortschatz niedergeschlagen: so meint gasconnade eben 'Aufschneiderei' oder 'Angeberei'). ${ }^{22}$ Es ist somit klar, daß der vom Gaskogner im gegebenen Beispiel behauptete Grund nicht der wirkliche Grund für sein Verhalten ist.

Zur gleichen Klasse gehört auch die Begründung Fladberts in Madame Bovary: ${ }^{23}$

(7) Grâce à ces travaux préparatoires, il (= Charles) échoua complètement à son examen d'officier de santé.

Da wir aufgrund des Kontextes wissen, daß die Vorbereitungen von Charles vor allem in ausgiebigem Besuch von Cabarets und nächtelangem Dominospiel bestand, können wir natürlich leicht die ironische Absicht FLAUBERTs erkennen.

Um noch ein Beispiel für eine mittels eines indirekten Sprechaktes vollzogene Ironie zu geben: wenn man zu Eltern sagt: "Ihre Kinder haben aber wirklich eine gesunde und frische Farbe», so macht man damit indirekt ein Kompliment. Ein geschickter Ironi- 
ker kann nun mit derselben Äußerung - ohne die Intonation zu verändern - ein Nicht-Lob, ja in manchen Fällen sogar einen Tadel evozieren. Das ist etwa in einer Situation, wie der in (8) beschriebenen der Fall:

(8) Gastgeberin: "Also, ich meine, daß moderne Eltern, wenn sie sich streiten, ihre Kinder zum Spielen nach draußen schicken sollten. Wir machen das übrigens ja auch, wenn wir mal so'n kleines Geplänkel haben.»

Eine Stunde später kommen die Kinder heim; Gast: "Ihre Kinder haben aber wirklich eine gesunde und frische Farbe; man sieht gleich, daß sie oft in der frischen Luft sind".

Dieses Beispiel ist deshalb interessant, weil ja die direkte Behauptung "Thre Kinder haben aber wirklich eine gesunde und frische Farbe" wahr ist; falsch ist hier die aus dieser Äußerung erschließbare Folgerung, m. a. W., der Gast will kein Kompliment machen, sondern die Gastgeberin in ihrer 'bonne conscience' entlarven.

Damit kann ich die oben gegebene Definition der Ironie, nämlich: "Das Gegenteil des Gesagten zu verstehen geben» wie folgt präzisieren:

(9) In einer ironischen Handlung gibt der Sprecher dem Hörer zu verstehen, daß er das 'Gegenteil' des Gesagten oder des aus diesem Erschließbaren meint.

('Gegenteil' ist hier im oben präzisierten Sinn zu verstehen, d.h. als Signalisierung des Durchbrechens der Metakonvention der Aufrichtigkeit; anders gesagt: 'Gegenteil' meint nicht einfache Umkehrung des Gesagten, also etwa: "Nimm mein Lob als Tadel», sondern nur: "Nimm mein Lob als Nicht-Lob».) ${ }^{24}$

Die gegebenen Beispiele erlauben uns nun, ein weiteres wesentliches Moment der ironischen Handlung zu bestimmen: durchgängiges Merkmal dieser Beispiele ist ja, daß sich jede ironische Handlung gegen das Verhalten einer Person oder Institution wendet (so wird im Chirurgenbeispiel die Person oder Institution 'König' kritisiert; im Quintilianbeispiel sind dies die Einwohner von Tarragona und im letzten Beispiel ist dies die Gastgeberin). Diese entlarvende Kritik bezeichne ich als abstrakte Negativität; ${ }^{25}$ 'Negativität', weil der Ironiker das Verhalten einer Person kritisiert und diese in ihrer 'bonne conscience' und Selbstzufriedenheit infragestellt; 'abstrakt', weil der Ironiker dieses Verhalten nur negiert, ohne seinem Opfer irgendwelche konkrete und konstruktive Hinweise zu geben. Mit seiner abstrakten Negativität zieht der Ironiker seinem Opfer gleichsam 'den Boden unter den Füßen weg', aber: er läßt sein Opfer 'in der Luft hängen', ohne ihm dabei zu helfen, wieder 'auf die Erde zu kommen'. 
Um das am letzten Beispiel zu verdeutlichen: man kann annehmen, daß die Gastgeberin verblüfft ist, ja sogar, daß sie errötet; dies einfach deshalb, weil sie sich in ihrem Selbstverständnis, ja sogar in ihrer sozialen Identität und Integrität infragegestellt fühlen muß. Es ist wahrscheinlich, daß nach dieser abstrakten Kritik des Gastes ein Bruch in der Kommunikation stattfinden wird - die Gastgeberin wird das Thema wechseln oder sich intensiv mit ihren Kindern beschäftigen und später vielleicht ihrem Mann sagen, daß man einen solch taktlosen Menschen nicht mehr einladen sollte. Konstruktiv und taktvoll hingegen hätte sich der Gast verhalten, wenn er sich mit der Gastgeberin in einem vertraulichen 'tête-à-tête' über die Familie im Allgemeinen und Besonderen unterhalten hätte - die Gastgeberin hätte ihm dann wohl gebeichtet, daß sie mit ihrem Mann völlig zerstritten ist, was ihm wiederum erlaubt hätte, ihr verständnisvoll einen konstruktiven Vorschlag zur Veränderung der eingefahrenen Familiensituation zu machen.

Damit glaube ich, die ironische Handlung wie folgt bestimmen zu können: die ironische Handlung ist eine besondere Form der Kritik am Verhalten von Personen und/oder Institutionen; diese Kritik ist als abstrakte Negativität bestimmbar und wird durch eine spezifische Technik vorgebracht, nämlich durch ein Signalisieren des Durchbrechens der Metakonvention der Aufrichtigkeit.

Aus dieser Bestimmung der ironischen Handlung folgt nun weiter, daß man mit einer ironischen Handlung all die Effekte intendieren kann, die man auch mit einer direkten Kritik des Verhaltens von Personen und/oder Institutionen erzielen kann. Das von STEMPEL angenommene Ziel der ironischen Handlung - die '(lächerlich machende) Bloßstellung' ist nur eines der möglichen Ziele einer ironischen Handlung: man kann die Intention haben, mit einer ironischen Handlung einen andern zu verblüffen, zu erfreuen, zu belustigen, zu beleidigen, zu demütigen, bloßzustellen, ja sogar auch, ihn zu überzeugen.

Das ist auch von der klassischen Rhetorik betont worden; man unterscheidet dort u. a. den Asteismus - das ist die witzig-urbane Ironie, mit der man seinen Gesprächspartner auf kultivierte Art unterhalten will; oder den Charientismus - das ist die wohlwollende und taktvolle ironische Kritik; oder schließlich den Sarkasmus - das ist die bittere Ironie, die - im Wissen, daß man das Bestehende doch nicht verändern kann - den Gegner mit beißender und zugleich resignierender Schärfe bloßzustellen sucht.. ${ }^{26}$

Und da wir mit einer ironischen Handlung durchaus die ernsthafte Absicht verbinden können, den andern von der Falschheit seines Verhaltens abzubringen, konnte JANKÉĹ́vITCH zurecht bemerken:

"Le mensonge est en malice et en dol ce que l'ironie est en sympathie (...) Exprimer pour voiler, mais aussi voiler pour mieux suggérer; écrire pour être mécompris, mais finalement se faire mécomprendre 
pour convertir plus efficacement son prochain à ce qu'on croit être la vérité: voilà l'invisible visibilité, la transparente opacité du masque ironique, voilà cette intériorisation extériorisante qui est, du même coup, extériorisation intériorisante (. . . $)_{0.27}$

\section{EINIGE GEBRAUCHSWEISEN UND THEORIEN DER IRONIE}

Die bisherigen Erörterungen erlauben uns, verschiedene umgangssprachliche Gebrauchsweisen des Lexems Ironie wie auch einige Theorien über die Ironie abzuleiten. Da ich nicht den Anspruch erheben kann, alle Gebrauchsweisen und Theorien exhaustiv darzustellen, sei im folgenden nur das Prinzip dieser Ableitung verdeutlicht.

Aus dem bisher Gesagten folgt, daß sich Ironiker und Zuhörer gleichsam verdoppeln müssen. Das Ich des Ironikers spaltet sich ja in zwei Teile: der eine Teil spricht normal und aufrichtig - so wie es die Metakonvention der Aufrichtigkeit verlangt; der andere Teil aber stellt dieses normale kommunikative Verhalten zugleich infrage, da er zu verstehen gibt, daß das 'Gegenteil' des Gesagten gemeint ist. Dieses Verhalten bezeichne ich als parakommunikativ. Ebenso gilt für den Hörer, daß er sich - um mich auf das Schema (10) zu beziehen - in Hk und Hp spalten muß, d.h. er muß sowohl die kommunikativen Signale als auch die parakommunikativen 'Ironiesignale' verstehen.

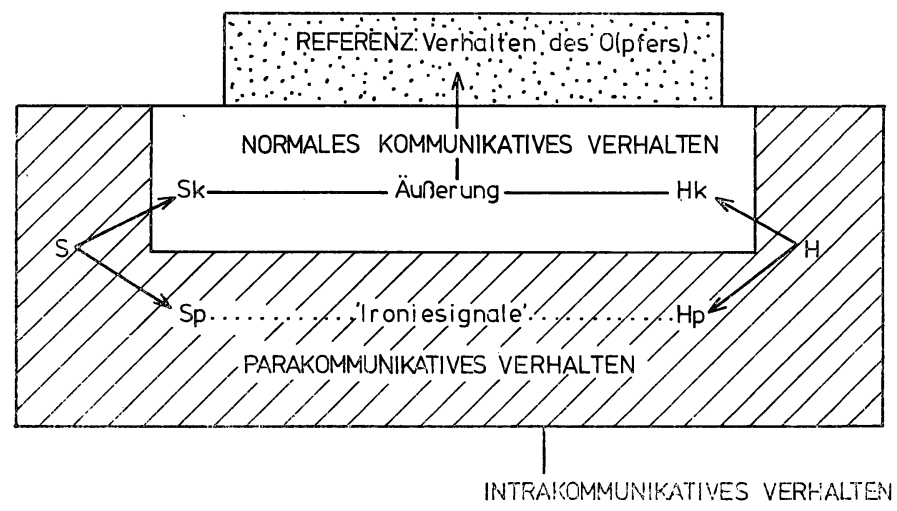

Das Schema läßt sich sehr gut durch einen Terminus verdeutlichen, den Friedrich SoHrEgre oft zur Kennzeichnung seiner Ästhetik und Philosophie verwendet hat, nämlich: Parekbasis (oder auch: Parekbase). ${ }^{28}$ Parekbasis ist ein terminus technicus aus dem griechischen Drama (oft auch einfach als Parabase bezeichnet) und meint jene Szene, in der 
mehrere Schauspieler als Chor nach vorne an die Bühnenrampe treten und sich direkt an das Publikum wenden; der Chor tritt somit aus dem dargestellten dramatischen Geschehen heraus (das ist die $E k$-basis), aber nicht so, daß er sich außerhalb und unbeteiligt neben dies Geschehen stellte; der Chor tritt vielmehr neben das dramatische Geschehen und zwar in dem Sinne, daß er dies Geschehen zugleich mitspielt und daneben kommentiert (das ist die Para-basis).

Deshalb kann F. ScHLEGEL auch metaphorisch sagen: "Ironie ist permanente Parekbase». Der ironischen Alltagshandlung und der dramaturgischen Parekbase ist ja eigentümlich, daß die Sprecher aus dem je normalen Geschehen heraustreten, sich neben dieses Geschehen stellen, ohne aus der Kommunikation herauszutreten. Sie unterscheiden sich dadurch, daß eben im Falle der Parekbase das normale und erwartete Verhalten sich in der 'Mllusionswelt: dramatisches Geschehen' abspielt, während bei der ironischen Alltagshandlung das erwartete Verhalten in der 'Realwelt' zu situieren ist. Daß die ironische Handlung ein intrakommunikatives Verhalten ist, kann man deshalb auch am Phänomen der 'Tllusionszerstörung' oder der 'literarischen Ironie'29 verdeutlichen: so tritt zwar A. GIDE, wenn er sich in den Faux-Monnayeurs direkt an den Leser wendet, aus der von ihm dargestellten Wirklichkeit heraus, aber er kann sich nicht 'außerhalb' der fiktionalen Wirklichkeit stellen die Faux-Monnayeurs mögen zwar ein ironischer Roman sein, aber sie sind und bleiben ein Roman.

Eine ironische Alltagshandlung ist offensichtlich dann gegeben, wenn die Rollen S, Sk und Sp im Schema (10) von einer Person und die Rollen $\mathrm{H}$, Hk und $\mathrm{Hp}$ von einer anderen Person, die gleichzeitig auch das Opfer der Ironie ist, verkörpert werden. Diese Normalform der Ironie bezeichne ich als direkte und unmittelbare Ironie.

Nun gibt es auch andere - abgeleitete - Erscheinungsformen der Ironie, die dadurch gekennzeichnet sind, daß einzelne Momente im Schema (10) von verschiedenen konkreten Personen verkörpert werden; oder in denen alle Momente durch einen einzigen Sprecher personifiziert werden. Das ist offensichtlich bei der Selbstironie der Fall: der Selbstironiker ist sich zugleich sein eigener Hörer und auch Opfer und verdoppelt sich sowohl als Sprecher als auch als Hörer. Von dieser monologischen Selbstironie ist die dialogische Selbstironie zu unterscheiden, in der ein Sprecher sich selbst einem andern gegenüber als Opfer darstellt (in diesem Fall verkörpert der Sprecher die Rollen des S, Sk, Sp und O, der Hörer hingegen nur die Rollen des H, Hk und Hp).

Das WEINRICHsche Ironiemodell, das ja von drei konkreten Sprechern ausgeht, personifiziert offensichtlich die Momente S, Sk und Sp zu einer Person, eben Sokrates; Euthyphron ist in diesem Modell die Personifizierung von Hk und (!) des Opfers der Ironie; wir als Leser nehmen die Rollen des Hp und des H ein.

Betont sei, daß diese WEINRICHsche Konzeption (wie auch andere Ironiemodelle, die ich hier nicht berücksichtigen kann) nicht abso- 
lut falsch ist, denn sie erklärt ja eine bestimmte Erscheinungsform der Ironie; aber: insofern als WeINRICH diese Erscheinungsform verallgemeinert und zum 'elementaren' Ironiemodell hypostasiert, ist seine Konzeption falsch; 'falsch' in dem Sinne, daß diese Konzeption die wesentlichen Momente der Ironie nicht bestimmen kann.

Um noch einige andere Erscheinungsformen der Ironie zu behandeln: objektive oder auch tragische Ironie ist dann gegeben, wenn ein Protagonist die Rolle des Sk einnimmt und sich aufrichtig, eben nach bestem Wissen und Gewissen verhält. Die Rolle des Sp wird von einer anderen Person eingenommen. Um das zu verdeutlichen: wenn mein Freund von einer langen Reise zurückkommt und mir sagt, daß er sich wahnsinnig freue, seine Freundin wiederzusehen, ich aber weiß, daß diese inzwischen mit einem anderen zusammenlebt, dann kann man wohl auch hier zurecht von 'tragischer' Ironie sprechen. In diesem Falle ist mein Freund Sk und O, ich aber nehme nicht nur die Rolle des Sp und des $H$ ein, sondern auch die des S, d.h. ich bin so etwas wie ein 'Großsubjekt', das über dem Laufe der Dinge steht. Dies erklärt auch, daß man bei der Bestimmung der tragischen Ironie oft auf 'Gott' oder die 'List der Vernunft' zurückgreift, welche die guten und rechtschaffenen Absichten der Handelnden allwissend zunichte machen. Nun kann man auch im Falle der literarischen Kommunikation von unmittelbarer, wenn auch indirekter, Ironie sprechen. In diesem Fall nimmt der Autor die Rollen von S, Sk und Sp ein und der Leser die des H, Hk und Hp. Aber diese gängige literaturwissenschaftliche Bestimmung der Ironie ist nur dann gerechtfertigt, wenn man die beiden folgenden Annahmen macht:

1. der Leser muß sich unmittelbar angesprochen fühlen; und dies wird der Fall sein, wenn er sich

2. mit den in der fiktionalen Wirklichkeit dargestellten Protagonisten, m. a. W., den Opfern der Ironie, identifizieren kann.

Der Leser verkörpert somit bezogen auf die Äußerungs- oder Lesesituation die Rollen von $\mathrm{H}$, Hk und $\mathrm{Hp}$; die Protagonisten hingegen die Rolle des $\mathrm{O}$. Aber: insofern als er sich mit einem Protagonisten in der dargestellten Wirklichkeit identifiziert, verkörpert er - wie in der direkten Ironie - die Rollen des Hk und des O denn er muß ja zugestehen, daß er genauso wie dieser Protagonist Opfer einer ironischen Handlung hätte werden können. 
Um das an FlaUberTs Education sentimentale zu verdeutlichen: Als Leser dieses Romans können wir über das Opfer der FLAUBERTschen Ironie, hier Frédéric, lächeln - verkörpern wir doch die Rollen des $\mathrm{H}, \mathrm{Hk}$ und des Hp. Wir können uns genauso allwissend wie der Autor FlaUbERT hinsichtlich der dargestellten Wirklichkeit fühlen. Denn: wenn für FlaUbERT gilt: "L'auteur, dans son œuvre doit être comme Dieu dans l'univers, présent partout et visible nulle part «, ${ }^{30}$ dann gilt auch für uns als Leser, daß FLAUBERT uns an dieser Allwissenheit teilhaben läßt oder uns zumindest das Gefühl gibt, allwissend über den Dingen des Lebens zu stehen und dieses Gefühl vermittelt uns FLAUBERT vor allem durch seine ironische (und damit auch versachlichende!) Darstellung. Aber: wenn wir uns in den überzogenen Träumen und utopischen Plänen von Frédéric, in seinen Ängsten und seiner Ziellosigkeit wiederentdekken, kurz: wenn wir uns mit Frédéric identifizieren, dann sind wir selbst Opfer der FladberTschen Ironie. ABER wir sind keine hilflosen Opfer, denn als allwissend gemachte Leser können wir uns selbst in ironischer Distanz belächeln. Und genau hier ist wohl auch das Emanzipatorische von guter Literatur zu suchen; und dies Emanzipatorische glaube ich hier durch eine leichte Variierung der KIERKEGaARDschen Formel umschreiben zu können, nämlich als: „Den Ernst solltest du in Zukunft nicht mehr so ernst nehmen". Um noch kurz auf die romantische Ironie einzugehen: romantische Ironie ist dann gegeben, wenn man die ironische Handlung zum Habitus macht und sich zum Dauerironiker 'hochstilisiert'. KrERKEGAARD hat das auf die kurze Formel gebracht: romantische Ironie ist unendliche absolute Negativität; ${ }^{31}$ 'absolut', weil dieser Dauerironiker alles Bestehende kritisiert und weil ihm »das gesamte Dasein (. . .) fremd " und er "wiederum dem Dasein fremd geworden ist«. ${ }^{31}$

Will man diese Haltung bewerten, so kann man sagen, daß der Dauerironiker subjektivistisch, ja sogar solipsistisch ist, weil er ja meint, sich allein über alle bestehenden gesellchaftlichen 'Spielregeln' hinwegsetzen zu können.

Deshalb ist diese habitualisierte Pose des Ironikers in bestimmtem Sinne auch parasitär, muß er sich doch, um überhaupt ironisch sein zu können, auf das normale kommunikative Verhalten stützen.

"Dauerironie ist - wie WELLFRSHOFF sagt ${ }^{32}$ - nur eine neue falsche Identität, die die eigene Leere unter der fadenscheinigen Maske angeblicher Reflektiertheit verbirgt».

Damit ist auch HeGELs Kritik der romantischen Ironie umschrieben: HEGEL kritisiert die zur Lebenshaltung und-philosophie gemachte Dauerironie der Romantiker, weil sie in abstrakter negativer Attitude stecken bleibt, ohne eine neue Sittlichkeit setzen zu können. Besonders 
heftig kritisiert HEGEL dabei F. ScHLEGEL und TIEOK, nachsichtiger geht er mit SolGER um. Und dem 'Ironiker' SoKRATES verzeiht er sogar, ${ }^{8}$ weil - so die geschichtsphilosophische Annahme HEGELS - SOKRATES ein 'Kind seiner Zeit' war. SokraTes konnte mit seiner 'abstrakten Moralität' nur die bestehende griechische Sittlichkeit destruieren, ohne aber selbst positive Alternativen setzen zu können. Und wenn HEGEL den Sokrates kritisiert, so tadelt er nicht die Person Sokrates, sondern dessen Philosophie: denn diese Philosophie destruiert nur die gängigen Verstellungen und Handlungsnormen, ohne uns zu sagen, wie wir uns in konkreten Einzelhandlungen verhalten sollen.

Dies scheint mir auch die gängige Bewertung des Ironikers zu sein: wir fühlen uns zu ihm hingezogen und doch zugleich von ihm abgestoßen, vor allem deshalb, weil er erstarrte Vorstellungen, falsche Autoritäten, ungerechtfertigte Machtverhältnisse und falsche Identitäten infrage stellt - und einige verachten ihn sogar in seiner Gestalt als 'Dauerironiker' - denn diesem gestehen sie nicht einmal mehr den Schein von Überlegenheit zu.

\section{GIBT ES IRONIESIGNALE?}

Abschließend muß ich noch auf die bisher ausgeklammerte Frage der Ironiesignale eingehen. QUINTILIAN hat dazu kurz bemerkt, daß die Ironie "aut pronuntiatione intellegitur aut persona aut rei natura ${ }^{33} \mathrm{~d}$. h., wir erkennen die Ironie entweder aufgrund prosodischer Gegebenheiten oder aufgrund unserer Kenntnis des Sprechers oder schließlich aufgrund unserer Kenntnis der behandelten Sache. Was man bei dieser Beschreibung unterstreichen muß, ist die Tatsache, daß die von QUINTIIIAN genannten 'Erkennungshilfen' der Ironie keine sprachlichen Signale im eigentlichen Sinne dieses Terminus sind - denn diese 'Erkennungshilfen' gehören ja dem Paraoder Extralinguistischen an. Und die Tatsache, daß wir zur Identifizierung einer ironischen Handlung auch unser Wissen über den Sprecher und die behandelte Sache zu Hilfe nehmen müssen, erklärt auch, daß ironische Handlungen oft nur für Eingeweihte 'entschleierbar' sind. Man kann deshalb der folgenden mit normaler Intonation vorgebrachten Äußerung:

(11) Sonntag war ich bei Müllers; es hat den ganzen Tag geregnet; na ja, der Fernsehempfang war wenigstens gut

die ironische Absicht nicht 'ansehen'. Aber (sofern (11) als ironisch intendiert ist): wie merken wir denn, daß der Ernst nicht ernst gemeint ist? Anders gesagt: wie 'signalisiert' uns ein Sprecher seine ironische Absicht? 
Um diese Frage exemplarisch am Beispiel (11) zu beantworten: Zunächst zum Gebrauch von wenigstens: mit wenigstens wird in Kontexten wie (11) eine relativ komplexe Argumentation vollzogen, die sich wie folgt umschreiben läßt. Wenn ein Sprecher einen negativ bewertbaren Sachverhalt wie etwa: "Das Auto hat keinen Komfort" äußert, so muß er - aufgrund seines Alltagswissens wie auch der Unterstellung, daß der andere dieses Wissen teilt - annehmen, daß sein Zuhörer folgert: "Also ist das Auto schlecht" (oder: "Also sollte ich dieses Auto besser nicht kaufen (I). Um ihn von diesem Schluß abzubringen, kann man einen positiv bewertbaren Sachverhalt formulieren, also etwa: "Das Auto hat keinen Komfort (p), na ja, der Motor ist wenigstens unverwüstlich (q)«. Durch den im wenigstens-Teilsatz repräsentierten Sachverhalt $q$ wird der vorher genannte Sachverhalt $p$ gleichsam kompensiert (anders gesagt: der Zuhörer steht vor der Entscheidung, ob er sich nicht doch dieses Auto kaufen sollte).

Wenn nun der im wenigstens-Teilsatz repräsentierte Sachverhalt von den meisten Sprechern wie auch vom Sprecher, der diese Äußerung vollzieht, positiv bewertet wird, läßt sich sagen, eine Äußerung wie: "Das Auto hat keinen Komfort; na ja, der Motor ist wenigstens unverwüstlich" ist kommunikativ kohärent verwendet. Mit 'kommunikativer Kohärenz' fasse ich somit QUINTIIIANs Kenntnis der Sache und des Sprechers wie auch unser (mehr oder weniger bewußtes) Wissen um sprachliche Argumentationsregeln zusammen. Betont sei, daß jede kommunikativ kohärente Äußerung notwendig auch sprachlich kohärent ist, $d$. h. notwendige Bedingung einer erfolgreichen Argumentation und Kommunikation ist, daß der Sprecher sich an die Aussprache-, Syntax- und Semantikregeln einer bestimmten Sprache hält wie auch, daß er seine Äußerungen situationsadäquat realisiert.

Wenn wir nun wissen, daß der Sprecher von (11) "Sonntag war ich bei Müllers; es hat den ganzen Tag geregnet; na ja, der Fernsehempfang war wenigstens gut" ein eingefleischte Gegner des Fernsehens ist, dann gilt, daß er seine Äußerung nicht kommunikativ kohärent vollzogen hat, d.h. im gegebenen Fall, daß er - eben weil er weiß, daß wir wissen, daß er das Gegenteil dessen sagt, was er meint - seine Äußerung ironisch verstanden wissen will. Um das zu verallgemeinern:

(12) Ein Sprecher teilt seinem Zuhörer seine ironische Absicht durch 'geschicktes' Durchbrechen der kommunikativen Kohärenz seiner Äuße- 
rung mit; und zwar derart, daß er ihm gleichsam sagt: 'Nimm das Gesagte oder das aus diesem normalerweise Erschließbare nicht so wie du es gewöhnlich gemäß der Metakonvention der Aufrichtigkeit tun wïrdest'.

Offensichtlich ist auch dann eine Verletzung der kommunikativen Kohärenz gegeben, wenn ein Sprecher durch ein 'geschicktes' Durchbrechen der pronuntiatio-Regeln seine ironische Absicht 'signalisiert' (also etwa, wenn er den Satz: „Dein Aufsatz ist wirklich interessant" mit einer ungewöhnlichen Intonation äußert bzw. mit einer zum Sinn dieser Äußerung nicht passenden Mimik begleitet).

Wenn nun aber gilt, daß die ironische Intention immer nur im Durchbrechen der normalen Realisierungsbedingungen einer bestimmten Sprechhandlung 'signalisiert' werden kann, dann stellt sich die Frage, ob man zurecht von Ironiesignalen ${ }^{34}$ sprechen kann. Denn die 'Bedeutung' der 'Ironiesignale' ist ja nicht durch ein Signalsystem oder einen Code, sondern immer nur relativ zu den für eine bestimmte Sprechhandlung je geltenden Realisierungsbedingungen bestimmbar. Anders gesagt: es gibt keine Signale der Ironie, sondern immer nur das Anzeigen des Durchbrechens der kommunikativen Kohärenz; und genau in diesem Anzeigen des Bruchs oder - wie wir auch sagen können - in diesem ernsten Spiel mit der Metakonvention der Aufrichtigkeit zeigt sich die ironische Intention.

Anschrift des Autors:

Prof. Dr. Ekkehard Eggs

Lehrgebiet Romanistik

Universität Hannover

Im Moore 21

D-3000 Hannover 1

\section{ANMERKUNGEN}

1 STEMPEL (76), S. 234.

2 Freud (70), S. 70; cf. auch Stemper (76), S. 215. Auffallend in der Untersuchung von STEMPEL (76) ist, daß sich STEMPEL bei seiner Erörterung der Ironie ausschließlich auf Witz(!)-Beispiele aus FrEUD (70) stützt - ohne diese beiden Formen des 'uneigentlichen' Sprechens voneinander zu trennen. In seiner Diskussion der Ironie bleibt STEMPEL (76) - trotz einiger kritischer Anmerkungen - der FrEUDschen Untersuchung zum Witz eng verpflichtet. Dazu ist zu sagen, daß FreUd (70) keine Abhandlung über die Ironie geschrieben hat (Freud (70) spricht nur kurz bei der Erörterung der Witztechnik: Darstellung des Gegenteils von der Ironie; cf. S. 71), sondern eine Abhandlung über die soziale Situation, in der man sich Witze erzählt. 
${ }^{3}$ Cf. dazu HASS/MoHrLÜDER (73). Trotz unterschiedlicher Bestimmung des Begriffs der Ironie ist allen Autoren der Teile I-IV in HASs/MOHRLÜDER (73) gemeinsam, daß sie sich als direkte Adressaten der in einem literarischen Werk dargesteliten ironischen Handlungen begreifen.

4 Zur Sokratischen Methode oder Ironie cf. die Darstellung in HEGEL (71), Bd. 18, S. 456ff und KTERKEGAARD (76).

5 Cf. etwa folgende Stelle: "So wird es demnach für mich, du bewundernswürdiger Euthyphron, wohl das Beste sein, daß ich dein Schüler werde . ..» (Platon (57), S. 182-5a).

6 WeINRICH (66), S. 62.

7 WeTNRTCH (66), S. 64.

8 WEINRICH (66), S. 65.

${ }^{9}$ HeGel (71), Bd. 11, S. 256. Der frühe HEGEL hat noch eine ähnliche Auffassung wie WEINRICH vertreten; cf. etwa die "Vorlesungen zur Geschichte der Philosophie» in: HeGer (71), Bd. 18, S. 457ff: „Daß er (= Sokrates) gewöhnliche Vorstellungen annimmt, sie sich geben läßt, hat die Erscheinung, daß er sich unwissend stellt, die anderen zum Sprechen bringt, - er wisse dies nicht; und nun fragte er mit dem Scheine der Unbefangenheit, es sich von den Leuten sagen zu lassen, sie sollen ihn belehren. Dieses ist dann die Seite der berühmten Sokratischen Ironie. Sie hat bei ihm die subjektive Gestalt der Dialektik, sie ist Benehmungsweise im Umgang; die Dialektik ist Gründe der Sache, die Ironie ist besondere Benehmungsweise von Person zu Person". MUECKE (69) nimmt in diesem Zusammenhang eine Art Zwischenposition ein, wenn er S. 87/8 bemerkt: "His (= Sokrates) ironic naivety is not that he presents himself falsely as knowing nothing but falsely as supposing he will learn something «.

${ }_{10}$ MUsIr (52), S. 280.

1 Cf. dazu zusammenfassend ROSEBerRY (74), insb. S. 7lff und S. 136ff. ${ }^{12}$ Dictionnaire du français vivant, éd. M. DavaU/M. Comen/M. Lallemand, Paris: Bordas, p. 669.

${ }_{13}$ Encyclopédie ou dictionnaire des Sciences, des Arts et des Métiers, T. 8, Paris 1765, p. 905; zur Geschichte des Ironiebegriffs BeHLER (72) S. 9ff. und Knox (73).

14 KierkegaARD (76), S. 244. KIERKegaARD schreibt dann ebd. weiter: $₫$ Eben damit aber ist die Ironie wieder aufgehoben. Es ist die allergewöhnlichste Form der Ironie, daß man mit ernster Miene etwas sagt, das doch nicht ernst gemeint ist. Die andere Form, daß man etwas zum Scherz, scherzend sagt, das ernst gemeint ist, kommt seltener vor. Indes wie gesagt, die ironische Redefigur hebt sich selber auf, sie gleicht einem Rätsel, für das man im Augenblick des Hörens die Auflösung hat». Diese Rede von der Aufhebung der Ironie ist dadurch zu erklären, daß KIERKEGAARD nicht genau zwischen Lüge und Ironie trennt (cf. S. 243 seine Bestimmung der Ironie: nihr Kennzeichen ist, daß man das Gegenteil dessen sagt, was man meint(). Diese fehlende begriffliche Trennung erklärt auch, daß KTERKEGAARD einen kontinuierlichen Ửbergang zwischen Ironie und Lüge annimmt; cf. etwa 245ff: „Und je mehr dem Ironiker das Täuschen gelingt, je besseren Fortgang seine Falschmünzerei hat, um so größer ist seine Freude. Diese Freude aber genießt er in völliger Einsamkeit, und es ist ihm gerade darum zu tun, daß niemand seinen Trug merkt» - dies ist offensichtlich eine exakte Beschreibung des Lügners, nicht aber des Ironikers !

${ }_{15} \mathrm{Cf}$. dazu die einschlägigen Handbücher von LAUSBERg (60), S. 302ff, Pletr (73), S. 93ff und Pletr (75), S. 262ff. Wir gehen etwas ausführlicher auf diese Behandlung der Ironie durch die Rhetorik ein, weil auch in neueren linguistischen Untersuchungen der Ironie der gleiche Status wie der Metapher zugeschrieben wird. So ist die Erklërung der Metapher und der Ironie bei EHRICH/SAILE (72), S. $276 f f$ - trotz modernerer Terminologie - völlig identisch mit der rhetorischen Theorie; auch GRICE (75) behandelt die Ironie im Zusammenhang mit der Metapher (cf: S. 53) und zwar als Beispiele für die Verletzung der Maxime der Qualität. Diese lautet: "Try to make your contribution one that is true» und läßt sich nach GRICE in zwei besondere Teil. 
maximen aufgliedern, nämlich: $₫ 1$. Do not say what you believe to be false. 2. Do not say that for which you lack adequate evidence» (Grice (75), S. 46). Zu dieser Konzeption cf. unten Anm. 17

${ }_{16}$ Beispiel aus QuINTILIAN (70), VI, 3, §77.

17 Dazu ausführlicher EGGS (76), wo wir den Lügner mit dem Falschspieler vergleichen (beide halten sich an die Konventionen des jeweiligen 'Spiels', verletzen aber die Metakonvention der Aufrichtigkeit); der Ironiker ist mit dem Spielverderber insofern vergleichbar als beide ja zeigen, daß sie das 'Spiel' nicht mehr ernsthaft spielen.

Die Maxime der Qualität von GRICe (75) (s. o. Anm. 15) entspricht offensichtlich dem, was wir als Metakonvention der Aufrichtigkeit bezeichnen. U. E. hat die Maxime der Qualität (bzw. der Aufrichtigkeit) einen fundamental anderen Status als die Maximen der Quantität, der Relation und der Art und Weise. So kann ein Sprecher durchaus die Maxime der Qualität verletzen (m. a. W., bewußt lügen), ohne dadurch das Kooperationsprinzip (cf. GRICE (75), S. 45: "Make your conversational contribution such as is required, at the stage at which it occurs, by the accepted purpose or direction of the talk exchange in which you are engaged «) zu mißachten - man lese dazu nur die Ửberlegungen zur Notlüge in der Moralphilosophie oder mache sich einige Gedanken über die Bedingungen, unter denen Verhöre und Folter stattfinden. Ebenso ist es etwas völlig anderes, ob jemand zu dunkel, unklar oder zu ausführlich redet (d.h.: die Maxime der Art und Weise verletzt) oder ob jemand einen anderen bewußt täuscht (d. h.: die Maxime der Qualität verletzt), um ihn zu übervorteilen. Im ersten Fall nämlich kann man den andern bitten, sich klarer und kürzer auszudrücken, im zweiten Fall aber ist man 'existentiell' betroffen.

Die gleiche Behandlung der Maxime der Qualität, genauer: der Metakonvention der Aufrichtigkeit, und der übrigen Maximen (die ja die Konventionen umschreiben, wie man sich sprachlich zu verhalten hat) hat nun zur Folge, daß GRICE weder dem Phänomen der Ironie noch dem der Metapher gerecht werden kann. Zunächst zur Ironie: bei der Ironie wird zwar die Maxime der Qualität/Aufrichtigkeit durchbrochen, zugleich aber angezeigt, daß diese Maxime durchbrochen wird; deshalb kann man mit JANKÉLÉVITCH (50), S. 154 auch sagen: "L'ironie est encore plus sérieuse que le sérieux". Zur Metapher: wenn GRIOE das metaphorische Sprechen als Verletzung der Maxime der Qualität/Aufrichtigkeit begreift (und d.h. ja nichts anderes als: metaphorisches Sprechen ist unaufrichtiges Sprec hen), dann folgt er - wie ich verkürzend sagen möchte - einer langen 'positivistischen' Tradition, die da meint, daß nur exaktes, eindeutiges und 'widerspruch-freies' Sprechen zur 'Wahrheit', 'Wissenschaft' und auch zum 'korrekten Alltagssprechen' führt. Nun ist metaphorisches Sprechen sicherlich ein Abweichen vom normalen Sprachgebrauch, was aber nicht impliziert, daß dieses Sprechen falsch oder unaufrichtig ist; ja, man kann mit Metaphern durchaus wahr sprechen und seinen Zuhörern eine neue Erkenntnis vermitteln (in EGGS (78), S. 172ff habe ich versucht, diese andere Erklärung metaphorischen Sprechens ausführlicher zu begrün den).

${ }_{18} \mathrm{Zu}$ den Bedingungen des Gelingens von indirekten Sprechakten cf. SEARLE (75) und Franok (75).

19 Man könnte gegen die vorgeschlagene Klassifikation einwenden, daß all diese Sprechweisen 'normal' sind, weil sie ja alle in der Alltagskommunikation falktisch vorkommen. Dieser Einwand ist berechtigt, verfehlt aber die Pointe dieser Klassifikation. Denn: jeder Sprecher ist in der Lage zu bestimmen, ob die faktisch vorkommenden Äußerungen gewöhnlich oder in einem bestimmten Sinne ungewöhnlich sind. Sprecher (wie auch Linguisten) mögen sich zwar darüber streiten, ob ein bestimmter Sprachgebrauch normal oder nicht mehr normal ist (z. B.: "Rutsch mir den Buckel runter" oder: "Dein Verhalten hat mir das Herz zerrissen «), aber: all dies heißt eben nicht, daß sie nicht in der Lage sind, gewöhliches von ungewöhnlichem Sprechen zu unterscheiden, sondern nur, daß sie eine andere Vorstellung oder ein anderes Modell von dem, wie man sich normalerweise ausdrückt, haben. 
Um einem möglichen Mißverständnis vorzubeugen: man könnte ja - sich auf den umgangssprachlichen Sprachgebrauch stützend - gegen diese Klassifikation und Erklärung der ironischen Handlung weiter einwenden, daß auch ironisches Sprechen indirektes Sprechen ist, da ja der Sprecher 'nicht direkt' sagt, was er meint. Dieser Einwand ist insofern berechtigt, als Ironie ja auch ein 'uneigentliches Sprechen' ist; aber: Ironie ist kein indirektes Sprechen (im Sinne der hier vorgeschlagenen Terminologie); indirektes Sprechen zeichnet sich nämlich dadurch aus, daß von einem sprachlich artikulierten Illokutionstyp $X$ immer positiv auf einen anderen Illokutionstyp $Y$ geschlossen werden kann (also etwa: eine Behauptung wird vom Sprecher als Aufforderung intendiert und vom Hörer als solche erschlossen). Bei der Ironie hingegen ist ein solcher positiver Schluß nicht möglich, da der Ironiker seinem Zuhörer ja nur 'signalisiert': "Nimm meine Äußerung (oder das aus ihr Erschließbare) nicht so wie du es normalerweise tun würdest». Deshalb kann man auch indirekte Sprechakte in ironischer Intention verwenden (cf. dazu unten Beispiel (8)).

20 JANKÉLÉviTCH (50), S. 154.

21 Das Beispiel ist aus Jean PaULs "Vorschule der Ästhetik»; cf. dazu auch Preisendanz (70), S. 22.

${ }_{22}$ Zum Gaskogner' als 'Ethnotyp' cf. MarTy (77).

23 FlaUBERT: Madame Bovary, Paris: Classiques Garnier 1957, S. 10.

${ }^{24} \mathrm{Um}$ das an der folgenden Figur zu verdeutlichen:

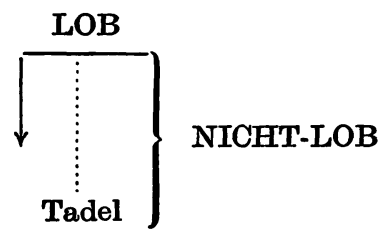

Durch eine ironische Handlung wird nur angezeigt, daß z. B. ein Lob als Nicht-Lob zu verstehen ist; ob dieses NICHT-LOB vom Hörer als Tadel, als destruierende oder als wohlwollende Kritik, als feinsinnige Anspielung oder als Bloßstellung erfahren wird, hängt vom jeweiligen Kontext ab.

${ }^{25}$ Diesen Terminus übernehme ich von KIERKRGAARD (76)- cf. unten Anm. 31. Cf. auch Stremper (76), der S. $217 f$ ebenfalls von 'Negativität' spricht. ${ }^{26}$ Cf. Puett (73), S. 95ff.

${ }^{27}$ JANKÉLÉVITCH (50), S. 52.

${ }^{28}$ Dazu HetMrRich (73).

${ }^{20}$ Zur 'literarischen' oder 'Fiktionsironie' cf. WEINREIOH (61) und WARNING (76), S. 311ff.

${ }^{30}$ Zur Flaubertschen Ironie cf. WAGNer (72) und besonders KopPe (77). 31 Cf. KrERKegaARd (76), S. $255 f$ f.

${ }^{32}$ W ELLERSHOFF (76), S. 424.

${ }^{33}$ QUINTILIAN (70), VIII, 6, §54.

${ }^{34}$ Cf. auch W ARNING (76a), S. 420: "Ironiesignale verfügen über kein eigenes System, über keinen eigenen Code, sondern sie operieren parasitär auf den Faktoren, die an dem jeweiligen Sprechakt beteiligt sind. Sie können prinzipiell an jedem dieser Faktoren ansetzen, um von seiner Störung her den ganzen Sprechakt zu stören und ihn damit - als einen ironischen - gelingen lassen. Ironiesignale, das wäre unser erstes Fazit, sind nicht rekurrent, sondern okkurent, d.h. bestimmbar nur unter den Bedingungen des je realisierten Sprechakts». Da die 'Anzeichen' der Ironie nur 'okkurent' sind, d.h. keine Signale im eigentlichen Sinne dieses Terminus, ist es m. E. sinnvoller, den Terminus 'Ironiesignal' zu vermeiden. 


\section{LITERATURHINWEISE}

Allemann, B., 1973.

Robert Musil: Ironie und Utopie des gegebenen Zustandes, in: Hass/ MOHRLÜDER 73, 257-263.

Воотн, W. C., 1974. $A$ rhetoric of irony, Chicago: The University of Chicago Press.

BEHLER, E., 1972.

Klassische Ironie, Romantische Ironie, Tragische Ironie, Darmstadt: Wiss. Buchgemeinschaft.

CoLe, P./Morgan, J. L. (eds) Syntax and Semantics, Vol. 3, "Speech Acts», London: Academic Press

EGGs, E., 1976.

"Täuschen: Eine semantisch-pragmatische Analyse», in: Linguistik und Didaletik 28: 313-326.

EGGS, E., 1978.

Die Rhetorik des Aristoteles. Ein Beitrag zur Argumentationstheorie und zur Syntax von komplexen Sätzen, Frankfurt: P. Lang (im Erscheinen)

EHRICH, V./SaIIE, G., 1972.

"Über nicht-direkte Sprechakter, in: WUNDERLICH, D. (Hrsg.): Linguistische Pragmatik, Frankfurt/M. Athenäum, 255-287.

Franck, D., 1975.

"Zur Analyse indirekter Sprechakte", in: EHRICH, V./FINKE, P. (Hrsg.): Beiträge zur Grammatik und Pragmatik, Kronberg: Skriptor, 219-231.

FreUd, S., 1970.

Der Witz und seine Beziehung zum Unbewußten, in: FreUd: Studienausgabe IV, Frankfurt/M.: S. Fischer, 9-219.

GrICE, H. P., 1975.

"Logic and conversation火, in: COLE/MORGAN (75): 41-58.

HASS, H.-E./MOHRLÜDER, G.-A, 1973 (Hrsg.): Ironie als literarisches Phänomen, Köln: Kiepenheuer \& Witsch.

HEGEL, G. W. F., 70.

"Solgers nachgelassene Schriften und Briefwechsel", in HEGEL: Theorie Werkausgabe: Suhrkamp, Bd. 11: 205ff.

HeGEL, G. W. F. 70.

Vorlesungen über die Geschichte der Philosophie I, in: HEGEL: Theorie Werkausgabe: Suhrkamp, Bd. 18.

HETMRICH, B. 1973.

"Der Begriff der Parekbase in der Ironie-Terminologie Friedrich Schlegels", in: HASS/MOHRLÜDER, 73: $163-167$.

JANKÉLÉVITCH, V., 1950.

L'ironie ou la bonne conscience, Paris: PUF.

KIERKEGAARD, S., 1976. Über den Begriff der Ironie, Frankfurt/M.: Suhrkamp.

KNox, N., 1973.

"Die Bedeutung von 'Ironie': Einführung und Zusammenfassung", in: HASS/MOHRLÜDER (73), $21-30$.

KOPPE, F., 1977.

Literarische Versachlichung, München: Fink.

LAUSBERG, H., 1960.

Handbuch der literarischen Rhetorik, 2 Bde, München: M. Hueber.

MARTY, J., 1977.

"Conflits linguistiques et ethnotypes occitans dans le théâtre français du XVII' siècle», in: Lengas 1: 41-48.

MUECKE, D. C., 1969.

The Compass of Irony, London: Meuthen \& Co Ltd.

MUEOKE, D. C., 1970.

Irony, London: Meuthen \& Co Ltd.

MUSIL, R. 1952.

Der Mann ohne Eigenschaften, Reinbek: Rowohlt 
Platon, 1957

"Euthyphron", in: Platon, Ges. Werke I, Reinbek: Rowohlt, 177-195.

Plett, H. F., ${ }^{2} 1973$.

Einführung in die rhetorische Textanalyse, Hamburg: Buske.

Plete, H. F., 1975.

Textwissenschaft und Textanalyse, Heidelberg: UTB.

Preisendanz, W., 1970.

Über den Witz, Konstanz: Universitätsverlag.

Preisendanz, W./Warning, R., 1976 (Hrisg.)

Das Komische, München: Fink.

QUINTILIAN, 1970.

M. Fabi Quintiliani: Institutionis oratoriae libri duodecim, ed. M. Winterbottom, 2 vol., Oxford.

ROSEBERRY, R. L., 1974.

R. Musil. Ein Forschungsbericht, Frankfurt: Fischer Athenäum.

SeARLE, J. R., 1975.

"Indirect Speech Acts", in: Cole/Morgan (75): 59-82.

STEMPEL, W.-D., 1976.

"Ironie als Sprechhandlung", in: Preisendanz/Warning (76): 205-235.

WAGNER, B., 1972.

Innenbereich und Äußerung, München: Fink.

WARNING, R., 1976.

"Elemente einer Pragmasemiotik der Komödie», in: Preisendanz/WarNING (76): $279-334$.

WARNING, R., 1976a.

"Ironiesignale und ironische Solidarisierung", in: Preisendanz/Warning (76): $416-423$.

WEINRICH, H., 1961.

"Fiktionsironie bei Anouilh", in: Literaturwiss. Jb., N. F., 1/2: 239-253

WEINRICH, H., 1966.

Linguistik der Lüge, Heidelberg: Lambert Schneider

WELLERSHOFF, D., 1976.

"Schöpferische und mechanische Ironie», in: Preisendanz/WarnING (76), $423-425$. 
